\title{
Intravitreous Delivery of Ab-Crystallin Ameliorates N-Methyl-N-Nitrosourea Induced Photoreceptor Degeneration in Mice: An in vivo and ex vivo Study
}

\author{
Ye Tao ${ }^{a, b}$ Lei Ding Anhui Yao $^{d}$ Zhen Yang ${ }^{d}$ Qinghua Yang ${ }^{b}$ Limin Qin ${ }^{b}$ \\ Linjun Yu ${ }^{d} \quad Y^{2}$ ling Gao ${ }^{d} \quad$ Yi Fei Huang ${ }^{b}$ Zhengwei Lie ${ }^{2}$ Dengke Teng ${ }^{f}$
}

\begin{abstract}
aDepartment of Physiology, Basic Medical College, Zhengzhou University, Zhengzhou, bepartment of Ophthalmology, Key Lab of Ophthalmology and Visual Science, General Hospital of Chinese PLA, Beijing, 'Department of Radiology, China-Japan Union Hospital of Jilin University, Changchun, dDepartment of Neurosurgery and Institute for Functional Brain Disorders, 153 Hospital of Chinese PLA, Zhengzhou, eDepartment of Neurosurgery, Zhongnan Hospital of Wuhan University, Wuhan, 'Department of Ultrasound, China-Japan Union Hospital of Jilin University, Changchun, China
\end{abstract}

\section{Key Words}

Retinitis pigmentosa $\cdot$ Photoreceptor $\cdot$ Apoptosis $・$ Crystallin

\begin{abstract}
Background/Aims: $\alpha \mathrm{B}$-crystallin $(\alpha \mathrm{BC})$ belongs to the family of small heat shock proteins that are necessary for maintaining oxygen homeostasis. This study was designed to explore the possible effects of $\alpha B C$ on $N$-methyl- $N$-nitrosourea (MNU) induced retinal degeneration and the underlying mechanisms. Methods: The $\alpha B C$ was injected into the vitreous bodies of MNU administered mice. The retinal morphology and visual function of experimental animals were analyzed by electroretinography (ERG), Spectral domain optical coherence tomography (SD-OCT), fundus photographs, optokinetic testing and immunohistochemistry assay. Results: Optokinetic behavioural tests and ERG examination suggested that the visual impairments of the MNU administered mice were ameliorated effectively by $\alpha B C$ treatment. OCT analysis showed that the major retinal architecture of the MNU administered mice was efficiently rescued by $\alpha B C$ treatment. Fundus examination suggested that the lesion size of the MNU administered mice was decreased by $\alpha B C$ treatment. MNU induced photoreceptor loss was also mitigated by $\alpha B C$ treatment as shown by hematoxylin and eosin staining. In particular, the immunostaining study suggested that $\mathrm{M}$-cone photoreceptors, rather than the S-cone photoreceptors, were preferentially rescued, indicating that the photoreceptor populations have different sensitivities to $\alpha B C$. The mechanism study suggested that the anti-apoptotic, anti-oxidative and neurotrophic function of $\alpha B C$ collectively contributed to these therapeutic

Y. Tao and L. Ding contributed equally to this work.

\begin{tabular}{ll}
\hline Prof. Dengke Teng, & Department of ultrasound, China-Japan Union Hospital of Jilin University \\
Prof. Zhengwei Li & Changchun, Jilin, 130000 (China) \\
And Prof. Yi Fei Huang & Tel. 86-13-678130561, Fax 86-13-678130566, E-Mail drdengketeng@163.com; xiulilidr@163.com
\end{tabular}
\end{abstract}




\section{Cellular Physiology Cell Physiol Biochem 2018;48:2147-2160 \\ \begin{tabular}{l|l|l} 
and Biochemistry Publis.1159/000492557 & $\begin{array}{l}\text { (c) } 2018 \text { The Author(s). Published by S. Karger AG, Basel } \\
\text { www.karger.com/cpb }\end{array}$
\end{tabular} \\ Tao et al.: Ab-Crystallin Ameliorates Photoreceptor Degeneration.}

effects. Conclusion: Intravitreal delivery of $\alpha B C$ could alleviate MNU induced photoreceptor degeneration and visual impairment. Further refinement of the $\alpha B C$ induced protection would afford a novel therapeutic strategy for retinitis pigmentosa.

(C) 2018 The Author(s)

Published by S. Karger AG, Basel

\section{Introduction}

Retinitis pigmentosa (RP) comprises a heterogeneous group of inherited retinal dystrophies that are characterized by progressive photoreceptor apoptosis. Millions of patients are affected by this ophthalmological disease and eventually become blind $[1,2]$. At present, therapeutic approaches for RP remain unsatisfactory owing to the complex etiologies. Therefore, novel strategies are necessary to prevent retinal degeneration as well as visual impairment. Accumulating evidence suggests that divergent mutations cause different RP phenotypes, and these mutations eventually lead to photoreceptor apoptosis $[3,4]$. This common cell death mechanism would provide a therapeutic target that is independent of the initial genetic defects. In particular, the oxidative stress plays a pivotal role in initiating photoreceptor apoptosis in RP. This notion is further supported by the fact that multiple antioxidants suppress the photoreceptor degeneration in RP models [5, 6]. N-Methyl- N-nitrosourea (MNU), a direct-acting alkylation toxicant, causes DNA lesions and paralyses the excision repair machinery within the cell nucleus, thereby inducing photoreceptor apoptosis in the mammalian retina [7]. After a single systemic administration, active signs of retinal degeneration, such as reduced outer nuclear layer(ONL) width, degraded electroretinogram (ERG) response, and hyper-expression of apoptotic labeling, occur in mice within 1 week after MNU administered. These characteristics faithfully mimic the pathological events taking place in RP. Therefore, the MNU administered animal has been recognized as a chemically induced RP model with rapid progressive dynamics [8-10]. Given its high reproducibility and convenience, the MNU induced RP animal model is widely utilized in pathological and therapeutic investigations.

Crystallin is a group of highly abundant proteins that are spatially organized in the ocular lens [11]. The $\alpha \mathrm{B}$-crystallin $(\alpha B C)$ is also expressed in the central neural system and retina as well as the lens. $\alpha B C$ belongs to the family of small heat-shock proteins and acts as a molecular chaperone during multiple cellular processes, including development, neurodegeneration, apoptosis and regenerative tissue repair $[12,13]$. The $\alpha B C$ induced beneficial effects in several human diseases have received considerable attention. However, its therapeutic potential in retinal degeneration is beginning to emerge. Recent studies have shown that the $\alpha \mathrm{BC}$ is involved in retinal pathologies, as $\alpha \mathrm{BC}$ expression is significantly up-regulated in the damaged retina [14]. Furthermore, $\alpha B C$ is deposited in the drusen of age-related macular degeneration (AMD). This alteration is considered to be an adaptive or compensatory response during AMD progression $[15,16]$. In therapeutic trials, over expression of $\alpha \mathrm{BC}$ in retinal pigment epithelium (RPE) cells could enhance cellular resistance against apoptosis. Conversely, deprivation of $\alpha \mathrm{BC}$ would render the RPE cells more susceptible to oxidative and endoplasmic reticulum(ER) stress [17].The cytoprotective ability of $\alpha B C$ is also demonstrated in photoreceptor cultures(e.g., 661W cells) [18]. Moreover, intravitreal injection of $\alpha B C$ results in axon regeneration in rats after acute optic never injury [19]. A recent proteomic analysis of the vitreous-retinal complex found that $\alpha B C$ contributed significantly to vitreoretinal pathologies [20].However, much of the aforementioned data are derived from cell culture studies, which cannot be readily translated to RP models, because the mechanisms may differ between in vitro and in vivo experimental conditions.

In this study, we assessed the $\alpha \mathrm{BC}$ induced effects on the photoreceptor degeneration of MNU administered mice. The therapeutic agents were injected into vitreous body to determine whether this delivery pathway could alleviate the pathology in the posterior segment of the eye ball. Further mechanism studies might determine the possibility of developing a novel therapy for RP. 


\section{Cellular Physiology Cell Physiol Biochem 2018;48:2147-2160 \begin{tabular}{ll|l} 
and Biochemistry Published online: August 9, 2018 & $\begin{array}{l}\text { (c) } 2018 \text { The Author(s). Published by S. Karger AG, Basel } \\
\text { www.karger.com/cpb }\end{array}$
\end{tabular}

\section{Materials and Methods}

\section{Study design and animal models}

C57BL/6 mice (male, 8 weeks old) were purchased from Laboratory Animal Center of General Hospital of PLA (Beijing, China), and were housed in an air-conditioned laboratory (room temperature: 180 to 23日, humidity: $40 \%$ to $65 \%$ ) on a 12 -h light/dark cycle. The care and maintenance of the animals were performed in compliance with the Association for Research in Vision and Ophthalmology's Statement for the use of animals. The design of the treatment protocol was based on the basic pathological feature of animal model and previous pharmacological studies. Typically, the retinal degenerative process in MNU administered mice occurred within 7 days with a dose of $60 \mathrm{mg} / \mathrm{kg}$ [21, 22]. This administered dose was considered as the optimum dose for model construction and has been used in multiple therapeutic trials [22, 23]. MNU solution ( $5 \mathrm{mg} / \mathrm{ml}$ ) was prepared by dissolving reagent in physiological saline containing $0.05 \%$ acetic acid. In order to establish the retinal degeneration model, the mice received a single intraperitoneal injection of MNU (60mg/kg; Sigma;St.Louis,MO). The animals were randomly assigned to three groups: 1) normal control group: no pharmacological administration; 2)MNU group: intravitreal injection of $2 \mu \mathrm{l}$ phosphatebuffered saline(PBS) $2 \mathrm{~h}$ post MNU administration; 3) MNU $+\alpha B C$ group: intravitreal injection of $1 \mathrm{mg} \alpha \mathrm{BC}$ $2 \mathrm{~h}$ post MNU administration. The intravitreal delivery of reagent was performed as follows: $1 \mathrm{mg}$ purified $\alpha B C$ (U.S.Biological, Swampscott, MA, USA) was dissolved in $2 \mu$ PBS. Lidocaine $(0.1 \%)$ was applied topically to the eye surface to anaesthetize the cornea. The pupils were dilated with atropine $(1 \%)$ and phenylephrine hydrochloride(2.5\%) eye drops (Xing Qi, Shenyang, China). The needle of a micro syringe was inserted from the limbus at a $45^{\circ}$ angle into the vitreous cavity, and the reagent was slowly injected in. Half a minute after injection, the syringe needle was removed, and neomycin eye drops (Xing Qi, Shenyang, China) were applied to the injected eyes to prevent infection. Mice with postoperative complications including lens opacity, intraocular hemorrhage, and retinal injury were excluded from this study. The choice of $\alpha \mathrm{BC}$ dose was based on a previous study [24]. After intraperitoneal injection of MNU, experimental animals required a period of time to recover from trauma. To minimize their suffering, these mice were maintained in shielded cages for 2 hours to determine whether they had any clinical signs or systemic symptoms. If no adverse effect was evident in the MNU administrated animal, the intravitreal injection procedure was then conducted.

\section{SD-OCT examination}

One week after MNU administration, the animals were subjected to spectral domain optical coherence tomography (SD-OCT) examination. The pupils of mice were dilated with atropine $(1 \%)$ and phenylephrine hydrochloride (2.5\%) eye drops (Xing Qi, Shenyang, China). Mice were anesthetized with by an intraperitoneal injection of $1 \%$ sodium pentobarbital ( $2 \mathrm{ml} / \mathrm{kg}$, Lot No.p3761; Sigma-Aldrich Corp.) and sumianxin $(0.05$ ml, Jilin Shengda Animal Pharmaceutical Co., Ltd., Jilin, China). Subsequently, mice were transferred to the recording plane of the SD-OCT system (Bioptigen, Durham, NC). A corresponding box was centered on the optic nerve head with eight measurement points separated by $3 \mathrm{~mm}$ from each other. The SD-OCT crosssectional images were analyzed with the InVivo VueTM DIVER 2.4 software (Bioptigen, Inc, NC, USA). Neural retinal thickness for the examined eyes was compared at each point by measuring the distance from the vitreous face of the retinal ganglion cell (RGCs) layer to the apical face of the RPE layer.

\section{Fundus photographs}

After OCT examination, mice were transferred on a warming plane while it was still anesthetized. The pupils were dilated repeatedly with atropine(1\%) and phenylephrine hydrochloride $(2.5 \%)$ eye drops (Xing Qi, Shenyang, China) to avoid retraction. Retinal structure was visualized and the retinal image was captured with a small animal fundus camera machine (Genesis, Tokyo, Japan). Signs of retinal degeneration, including alterations of the RPE and retinal arteries, and presence or absence of pigmented dots were noted during the examination. 10 mice in each animal group were subjected to the fundus examination. Devastation scale and lesion size were assessed subjectively by an examiner on the basis of photographs from all the mice in each group. 


\section{Cellular Physiology Cell Physiol Biochem 2018;48:2147-2160 \begin{tabular}{ll|l} 
and Biochemistry Published online: August 9, 2018 & $\begin{array}{l}\text { (c) } 2018 \text { The Author(s). Published by S. Karger AG, Basel } \\
\text { www.karger.com/cpb }\end{array}$
\end{tabular}}

\section{ERG recording}

Mice were dark adapted overnight before ERG examination. Subsequently, mice were anesthetized and their pupils were dilated following the aforementioned protocol. We transferred the mice to the recording platform under dim red light. The cornea of the mouse was anesthetized with a drop of proxymetacaine $(0.5 \%)$. The RETIport system (Roland Consult, Germany) with custom-made chloride silver electrodes were applied in the recording process. A loop electrode was placed over the cornea to serve as the active electrode. Needle reference and ground electrodes were inserted into the cheek and tail respectively. A brief white flash (3.0 $\left.\mathrm{cd} \cdot \mathrm{s} / \mathrm{m}^{2}\right)$ was delivered from a Ganzfeld integrating sphere to stimulate the response. A band-pass $(1 \mathrm{~Hz}-300$ $\mathrm{Hz}$ ) was used to amplify the recorded signals. Totally 60 photopic responses and 10 scotopic responses were collected and averaged for a- and b-waves analysis.

\section{Optokinetic testing}

Light adapted visual acuities and contrast sensitivities were measured using a two-alternative forced choice paradigm as described previously [25]. Briefly, stepwise functions for correct responses in both the clockwise and counter-clockwise direction were used to determine the response threshold. The initial stimulus in visual acuity measurements was set as $0.200 \mathrm{cyc} / \mathrm{deg}$ sinusoidal pattern with a fixed $100 \%$ contrast. The initial pattern in contrast sensitivity measurements was set as $100 \%$ contrast, with a fixed spatial frequency of $0.128 \mathrm{cyc} / \mathrm{deg}$. All patterns were presented at a speed of $12 \mathrm{degrees} / \mathrm{s}$ with the mean luminance of $70 \mathrm{~cd} / \mathrm{m}^{2}$. Visual acuities and contrast sensitivities of each mouse were measured for four times over a period of $24 \mathrm{~h}$.

\section{Histology assessment and Immunohistochemistry}

Mice were sacrificed and their eyecups were enucleated. Eyecups were immersed in a fixative solution containing 4\% paraformaldehyde (Dulbecco's PBS; Mediatech, Inc., Herndon, VA, USA) for 24 hours. Subsequently, the specimens were rinsed with PBS, dehydrated in a graded ethanol series, and embedded in paraffin wax. Five sections (thickness of $5 \mu \mathrm{m}$ ) were cut vertically through the optic nerve head of each eye. The sections were stained with hematoxylin and eosin (HE) and evaluated by light microscopy. Using the Image-Pro Plus software (Media Cybernetics, Silver Spring, MD), the adjacent thickness of the ONL was measured along the vertically superior-inferior axis at $250 \mu \mathrm{m}$ intervals. The mean ONL thickness of each mouse was averaged from five sections. Then sections were rinsed with $0.01 \mathrm{M}$ PBS, permeabilized with $0.3 \%$ Triton $\mathrm{X}-100$, and blocked with 3\% bovine serum albumin(BSA) for $1 \mathrm{~h}$ at room temperature. The peanut agglutinin(PNA) conjugated to Alexa Fluor 488 (1: 200, L21409, Invitrogen, USA), S-cone opsin, and M-cone opsin antibodies (1 : 400,Millipore, MA, USA) diluted in 0.1\% Triton X-100 and 1\% BSA in PBS. Then, sections were extensively washed with PBS, and incubated with Cy3-conjugated anti-rabbit IgG (1: 400, 711-165-152, Jackson ImmunoResearch Laboratories, PA, USA) and DAPI. Retinal sections were then rapidly rinsed five times with $0.01 \mathrm{M}$ PBS, and coverslipped with anti-fade Vectashield mounting medium (Vector Laboratories, Burlingame, CA, USA) for photographing.

\section{TUNEL assay}

Three days after MNU administration, the TUNEL (terminal deoxyuridine triphosphate nick-end labeling) assay was performed on paraffin sections to analyze the apoptotic status of the retinas. The in situ cell death detection POD Kit (Roche Diagnostics GmbH, Mannheim Germany) was used according to the manufacturer's protocol. The TUNEL sections were counterstained with DAPI, mounted on slides, and then visualized with confocal microscopy (LSM510, Zeiss, Oberkochen, Germany). The apoptotic index (AI) of ONL was calculated ([number of TUNEL-positive nuclei]/[total number of photoreceptor cell nuclei] $\times 100)$.

\section{Quantification of SOD activity and MDA content}

Three days after MNU administration, the animals were sacrificed and their eyecups were enucleated. Retinal tissue was added into the PBS containing 0.5\% Triton X-100(pH 7.4) and homogenized in ice cold by Grinders. Retinal tissue was centrifuged at $500 \times \mathrm{g}$ for 5 minutes at $4^{\circ} \mathrm{C}$. The suspension was assayed for protein contents to normalize enzyme activity. Superoxide dismutase (SOD) activity was examined with the SOD Assay Kit-WST (Jiancheng Biotech Ltd., Nanjing, China). A spectrophotometer with ultra-micro cuvettes 


\section{Cellular Physiology Cell Physiol Biochem 2018;48:2147-2160 \begin{tabular}{ll|l} 
and Biochemistry Published online: August 9, 2018 & $\begin{array}{l}\text { C } 2018 \text { The Author(s). Published by S. Karger AG, Basel } \\
\text { www.karger.com/cpb }\end{array}$ \\
\hline
\end{tabular} \\ Tao et al.: Ab-Crystallin Ameliorates Photoreceptor Degeneration.}

was used to measure the absorbance values. The content of malondialdehyde (MDA) was assessed using a total bile acids colorimetric assay under the guidance of the manufacturer's instructions (Jiancheng Biotech Ltd., Nanjing, China).

\section{Quantification of $\alpha B C$ levels in the retinas}

Thirty hours after MNU administration, the experimental animals were sacrificed. Their eyes were enucleated and dissected immediately. The neural retina was gently removed from the pigment epithelium layer of the eye-cup. Subsequently, western plot assay was performed to quantify the a BC protein levels in neural retinas of mice.

\section{Western blot analysis}

Retinas were cut into pieces and homogenized in buffer containing $0.23 \mathrm{~mol} / \mathrm{l}$ sucrose, $2 \mathrm{mmol} / \mathrm{l}$ EDTA, $5 \mathrm{mmol} / \mathrm{l}$ Tris-HCl (pH 7.5), and $0.1 \mathrm{mmol} / \mathrm{l}$ phenylmethylsulfonyl fluoride. After centrifugation, aliquot containing equal amounts of protein $(20 \mu \mathrm{g})$ were electrophoresed, transferred, and probed with polyclonal antiserum against STAT3 (Ser727, $1: 5000$, Cell Signaling Technology, MA, USA), or JAK2 (1:1000, Santa Cruz Biotechnology, TX, USA), or a rabbit anti- a BC (1:1000, Chemicon, CA, USA), anti-CNTF (1:1000, Santa Cruz Biotechnology, TX, USA), anti- BNDF (1:1000, Cell Signaling Technology, MA, USA), anti- pStat3 (1:1000, Cell Signaling Technology, MA, USA), anti-bax (1:1000, Santa Cruz Biotechnology, TX, CA), antibcl 2 (1:1000, Cell Signaling, MA, USA), anti-caspase3 antibody(1:1000, Cell Signaling Technology, MA, USA) or a monoclonal antibody against mouse $\beta$-actin (1:3000, Sigma-Aldrich, CA, USA). The membrane was washed thoroughly and then incubated with anti-rabbit or anti-mouse IgG horseradish peroxidase secondary antibodies (1:3000, Santa Cruz Biotechnology, CA). Bands were visualized using an enhanced chemiluminescence detection system (Super Signal West Pico Chemiluminescent Substrate Kit; Pierce, Rockford, IL, USA).

\section{Isolation of mitochondria and analysis of MnSOD}

Retinal tissue was washed and homogenized using buffer A [0.3 phenylmethylsulfonyl fluoride, 1 orthovanadate, $1 \mathrm{NaF}, 1 \mathrm{EDTA}, 10 \mathrm{Tris}-\mathrm{HCl}, 250$ sucrose (in mmol/l)], followed by serial centrifugations $(700 \times \mathrm{g}$, and $10000 \times \mathrm{g}$ for $30 \mathrm{~min}$ respectively ). The resulted supernatant was collected and defined as the cytosolic fraction, while the mitochondrial pellet was washed, collected and lysed in mitochondria specific buffer B [0.3 PMSF, 1 NaF,1 orthovanadate, 10 EDTA, 20 Tris- $\mathrm{HCl}, 150 \mathrm{NaCl}$ (in mmol/l), 1\% NP-40], followed by $10 \mathrm{~min}$ of centrifugation (21000 g). Then the supernatant was carefully collected and subjected to mitochondrial superoxide dismutase (MnSOD) analysis. MnSOD activity was measured using commercially available kits (Beyotime biotechnology, Shanghai, China)under the guidance of the manufacturer's instructions. MnSOD activity was expressed as relative percent of control.

\section{Quantitative real time-polymerase chain reaction ( $q R T-P C R$ )}

Total RNA was extracted from pooled retinal patches with a commercial reagent (Trizol, Gibco Inc., Grand Island, NY, USA), followed by cDNA synthesis using the $\mu$ MACS $^{\mathrm{TM}}$ DNA Synthesis kit (Miltenyi Biotech $\mathrm{GmbH}$, Bergisch-Gladbach, Germany). GAPDH was used as an internal standard of mRNA expression. Reactions were performed in a real-time CFX96 Touch PCR detection system (Bio-Rad Laboratories, Reinach, Switzerland). The amplification program consisted of polymerase activation at $95^{\circ} \mathrm{C}$ for $5 \mathrm{~min}$ and 50 cycles of denaturation at $95^{\circ} \mathrm{C}$ for $1 \mathrm{~min}$, annealing and extension at $59^{\circ} \mathrm{C}$ for 30 seconds. The primers used in qRT-PCR were: Bax:5'-AGCTCTGAACAGATCATGAAGACA3'(forward)and5'-CTCCATGTTGTTGT CCAGTTC ATC3' (reverse);Bcl-2:5'GGACAACATCGCTCTGTGGATGA3'(forward)and5'CAGAGACAGCCAGGAGAAATCAA 3'(reverse);Caspase-3:5'TGTCGATGCAGCTAACC3'(forward)and5'GGCCTCCACTGGTATCTTCTG3'(reverse); CNTF: 5'-CCT CTG TAG CCG TTC TAT CTG-3'(forward)and 5'- GGT TCT CTT GGA GTC GCT CT-3'; BNDF: 5'AAG GTG GAT GAG AGT TGA AG-3'(forward) and 5'-GAT GCT GGA AGG TAA TGT GT-3'(reverse). Relative expression levels were normalized and quantified to obtain the $\Delta \Delta \mathrm{CT}$ values (DATA assist Software v2.2, Applied Biosystems). 


\section{Cellular Physiology Cell Physiol Biochem 2018;48:2147-2160 \\ \begin{tabular}{ll|l} 
and Biochemistry Published online: August 9, 2018 & $\begin{array}{l}\text { (c) } 2018 \text { The Author(s). Published by S. Karger AG, Basel } \\
\text { www.karger.com/cpb }\end{array}$
\end{tabular}}

Tao et al.: Ab-Crystallin Ameliorates Photoreceptor Degeneration.

\section{Statistical analysis}

The statistical difference between the animal groups was processed using the ANOVA analysis followed by Bonferroni's post-hoc test. $P<0.05$ was considered as statically significant. The values are presented as mean \pm standard deviation.

\section{Results}

$\alpha B C$ levels in the neural retinas of experimental animals

Western plot was performed to quantify $\alpha \mathrm{BC}$ protein levels in the neural retina of animals in the three groups (Fig. 1.A). The retinal $\alpha \mathrm{BC}$ level was significantly higher in the $\mathrm{MNU}+\alpha \mathrm{BC}$ group than in the normal control group $(P<0.01 ; \mathrm{n}=10 ;$ Fig. $1 . B)$ and the MNU group $(P<0.01 ; \mathrm{n}=10)$. Moreover, the retinal $\alpha \mathrm{BC}$ protein level was not significantly different in the MNU group and the normal control group $(P>0.05 ; n=10)$. These findings suggest that the exogenously delivered $\alpha B C$ could reach the sensory retina. Intravitreal injection could act as an effective approach for $\alpha B C$ delivery.

\section{$\alpha B C$ induced protective effects on visual function}

The optokinetic behavioral test suggested that the MNU group responded extremely poorly to the stimulus: both visual acuity and contrast sensitivity of the MNU group were significantly lower compared with the normal control $(P<0.01 ; \mathrm{n}=10)$. Meanwhile, the $\mathrm{MNU}+\alpha \mathrm{BC}$ mice responded better than the MNU group: visual acuity and contrast sensitivity of the MNU $+\alpha B C$ group were both significantly higher compared with the MNU group $(P<$

Fig. 1. A. Western plot was performed to quantify the $\alpha \mathrm{BC}$ protein levels in the neural retina of mice. B. Retinal $\alpha \mathrm{BC}$ level in the $\mathrm{MNU}+\alpha \mathrm{BC}$ group was significantly increased than those in the normal control group and the MNU group.C. Optokinetic behavioral analysis suggested that mice in the MNU group responded poorly to stimulus. Visual acuity in the MNU group was significantly lower compared with the normal control group, whereas visual acuity in the $\mathrm{MNU}+\alpha \mathrm{BC}$ group was significantly higher compared with the MNU group. D. Contrast sensitivity in the $\mathrm{MNU}+\alpha \mathrm{BC}$ group was significantly higher compared with the MNU group, suggesting that optokinetic function

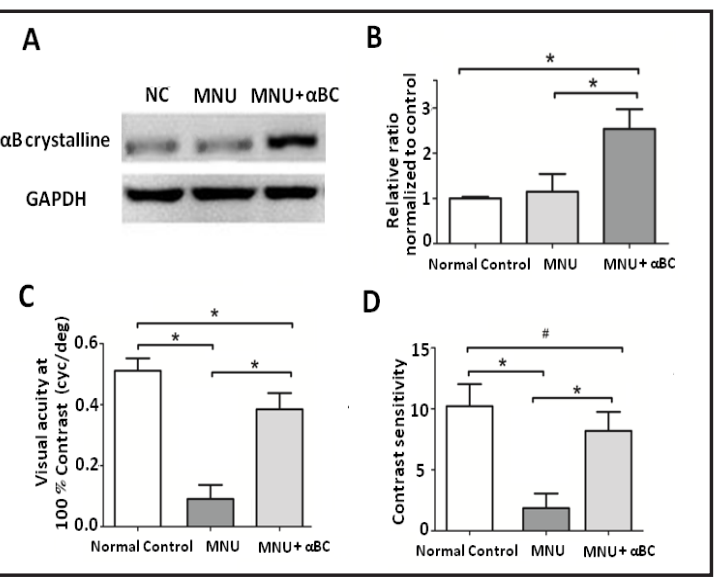
of MNU administered mouse was rescued by $\alpha \mathrm{BC}$ treatment (All the values were presented as mean \pm SD; ANOVA analysis followed by Bonferroni's post-hoc analysis was performed, ${ }^{*} \mathrm{P}<0.01$ for differences between groups; $\mathrm{n}=10$ ).

Fig. 2. A. Representative wave forms of both scotopic and photopic ERG responses. B. Photopic and scotopic b-waves in the MNU group were almost undetectable. However, the ERG function in the MNU $+\alpha B C$ group was less impaired: the amplitudes of photopic and scotopic b-wave in the MNU $+\alpha B C$ group were significantly larger compared with the MNU group (All the values were presented as mean \pm SD; ANOVA analysis followed by Bonferroni's post-hoc analysis was performed, ${ }^{*} \mathrm{P}<0.01$, for differences between groups; $\mathrm{n}=10$ ).

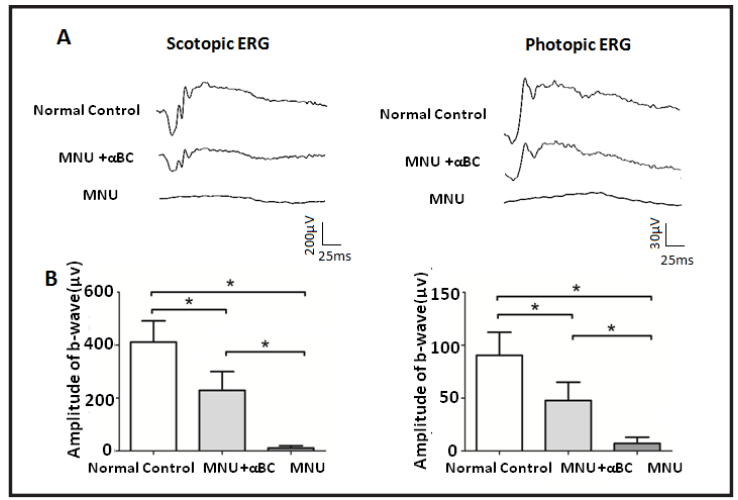


$0.01 ; \mathrm{n}=10$ ). Although visual acuity and contrast sensitivity of the MNU $+\alpha \mathrm{BC}$ group were lower compared with the normal controls (visual acuity: $P<0.01, \mathrm{n}=10$, Fig. 1.C; contrast sensitivity: $P<0.05, \mathrm{n}=10$, Fig. 1.D), these results suggeste that $\alpha \mathrm{BC}$ therapy could improve the optokinetic function of MNU administered mice. Subsequently, the experimental animals were subjected to ERG examination and the representative ERG wave forms of each group are shown in Fig. 2.A. As expected, both the photopic and scotopic b-waves of the MNU group were almost undetectable. However, the $M N U+\alpha B C$ group exhibited less impaired ERG function: both photopic and scotopic b-wave amplitudes of the MNU $+\alpha B C$ group were significantly larger compared with the MNU group $(P<0.01 ; \mathrm{n}=10$, Fig. 2.B $)$. It is noteworthy that photopic and scotopic b-wave amplitudes in the $\mathrm{MNU}+\alpha \mathrm{BC}$ group were respectively approximately $52 \%$ and $67 \%$ of those in the normal control group, indicating that cone function was preferentially rescued by $\alpha B C$.

\section{$\alpha B C$ induced protective effects on retinal morphometry}

In order to determine the $\alpha B C$ induced effects on retinal appearance, we photographed the mice using a fundus camera. Fundus photographs of the MNU group showed prominent damage due to MNU administration, as typified by the abundance of yellow-whitish lesions that spread throughout all retinal sectors (Fig. 3.A).In contrast, there were fewer lesions in the MNU $+\alpha B C$ group than in the MNU group. In particular, the lesions were devoid of pigments and were much smaller than the lesions in the MNU group. Subsequently, retinal thickness was assessed by SD-OCT examination in vivo (Fig. 3.B). The retinal organization of the MNU group was markedly disrupted by MNU administration. Retinal thickness was substantially reduced compared with the normal controls $(P<0.01 ; \mathrm{n}=10$, Fig. 3.C). Retinal thickness in the $\mathrm{MNU}+\alpha \mathrm{BC}$ group was also significantly reduced compared with the normal control group $(P<0.01 ; \mathrm{n}=10)$. Nevertheless, retinal thickness in the MNU+ $\alpha \mathrm{BC}$ group was significantly increased compared with the MNU group, indicating that MNU induced morphological impairment could be partially alleviated by $\alpha \mathrm{BC}$ treatment.

\section{$\alpha B C$ induced protective effects on photoreceptors}

The ONL thickness of HE stained sections was quantified to determine the $\alpha B C$ induced protective effects on photoreceptors. The ONL in the retinas of the MNU group disappeared after MNU administration. Conversely, a large proportion of the ONL was

Fig. 3. A. Fundus photographs showed that the retinal appearance in the MNU group was devastated markedly, as typified by the abundance of yellow-whitish lesions that spread throughout all retinal sectors. Conversely, there were fewer lesions in the MNU $+\alpha B C$ group compared with the MNU group. B. Mice were subjected to SD-OCT examination and their retinal thicknesses were quantified. C. Retinal thickness in the MNU group was significantly smaller than that in the normal controls. Retinal thickness in the $\mathrm{MNU}+\alpha \mathrm{BC}$ group was significantly larger compared with the MNU group. D. ONL in the retinal sections of the MNU group disappeared. A large proportion of ONL was preserved in the retina sections of $\mathrm{MNU}+\alpha \mathrm{BC}$ group. E. The mean ONL thickness in the MNU $+\alpha B C$ group was significantly larger compared with the MNU group. (All the values were presented as mean $\pm \mathrm{SD}$; ANOVA analysis followed by Bonferroni's post-hoc analysis was performed, $* \mathrm{P}<0.01$, for differences between groups; $n=10$; GCL: Ganglion cell layer; IPL: inner plexiform layer; INL: inner nuclear layer;

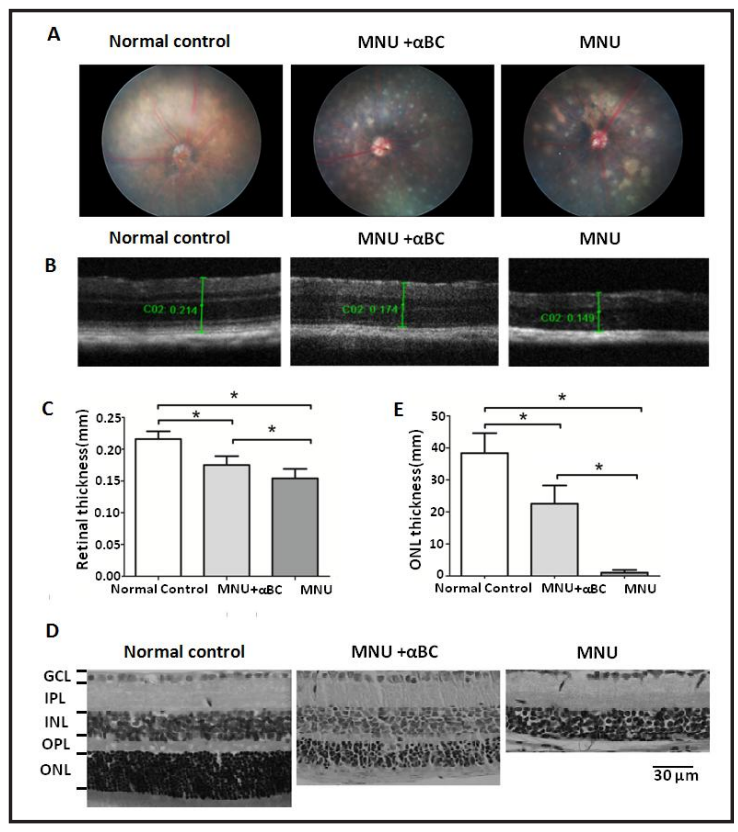
OPL: outer plexiform layer; ONL: nuclear layer).

\section{KARGER}


Fig. 4. TUNEL assay was performed to quantify the apoptotic activity in retinas. No TUNEL -positive cell was detected in the retinas of the normal control group. In contrast, many TUNEL -positive cells were found in the retinas of the MNU group. TUNEL-positive cells were also found in the retinas

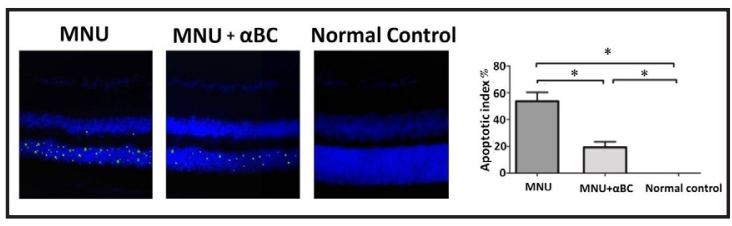
of the MNU $+\alpha B C$ group. The $\mathrm{AI}$ of the $\mathrm{MNU}+\alpha \mathrm{BC}$ group was significantly smaller compared with the MNU group $(\mathrm{P}<0.01 ; \mathrm{n}=10)$, suggesting that $\alpha \mathrm{BC}$ treatment could alleviate $\mathrm{MNU}$ induced photoreceptor apoptosis (All the values were presented as mean \pm SD; ANOVA analysis followed by Bonferroni's post-hoc analysis was performed, ${ }^{*} \mathrm{P}<0.01$, for differences between groups; $\mathrm{n}=10$ ).

Fig. 5. A. Immunostaining works on the retinal sections of mice from different groups. PNA staining was detected in the retinas of both normal control group and $\mathrm{MNU}+\alpha \mathrm{BC}$ group, but rarely in the MNU group. M-opsin staining was detected throughout the retina of the MNU $+\alpha B C$ group, although with a decreased intensity compared with the normal control

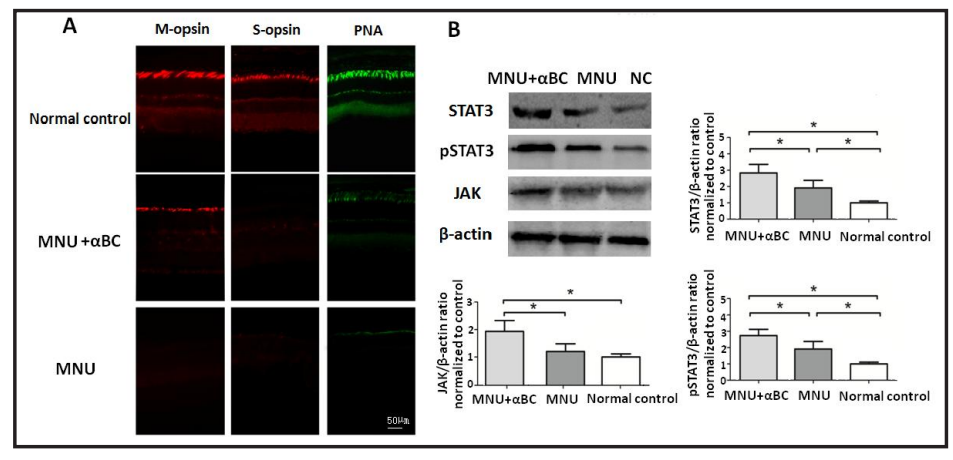
group. Of note, no S-opsin staining was found in the retinas of the MNU $+\alpha B C$ group. B. Protein levels of STAT3, phosphorylated STAT3 and JAK in the MNU $+\alpha B C$ group were significantly higher compared with the MNU group, suggesting that JAK/ STAT3 signal pathway was activated by $\alpha B C$ treatment. (All the values were presented as mean \pm SD; ANOVA analysis followed by Bonferroni's post-hoc analysis was performed, ${ }^{*} \mathrm{P}<0.01$, for differences between groups; $\mathrm{n}=10$ ).

retained in the retinas of the $\alpha \mathrm{BC}$ group (Fig. 3.D). The mean ONL thickness in the $\alpha \mathrm{BC}$ group was significantly increased compared with the MNU group $(P<0.01 ; \mathrm{n}=10$, Fig. 3.E). Furthermore, the TUNEL assay was performed to quantify the apoptotic activity in photoreceptors. A previous study had shown that the MNU induced apoptotic activation peaked at 3 days post administration(P3), and then returned to the baseline around P7 [22]. Therefore, we performed the TUNEL assay at P3 to capture the most evident changes (Fig. 4). No TUNEL -positive cell was detected in the retinas of the normal control group. In contrast, many TUNEL -positive cells were found in the retinas of the MNU group. In particular, most of the TUNEL-positive cells were concentrated in the ONL, suggesting that MNU toxicity induced massive photoreceptor apoptosis in mice retinas. TUNEL-positive cells were also detected in the retinas of the MNU $\alpha \mathrm{BC}$ group. However, the $\mathrm{AI}$ in the $\mathrm{MNU}+\alpha \mathrm{BC}$ group was significantly smaller than that in the MNU group $(P<0.01 ; n=10)$, suggesting that $\alpha \mathrm{BC}$ treatment could alleviate the MNU induced photoreceptor apoptosis. As rods account for the major photoreceptor population in murine retinas( $\sim 97 \%)$, the ONL should be considered as an indicator of rod vitality. Whether the minor population of cones $(\sim 3 \%)$ were effectively rescued by $\alpha B C$ treatment could not be deduced from HE stained sections. Consequently, we performed immunostaining to determine the vitality of cones(Fig. 5.A). Typical PNA staining was detected in both the normal control and MNU $+\alpha B C$ groups, but rarely in the MNU group. Specifically, we analyzed the immune fluorescence with $\mathrm{M}$ - and S-opsin antibodies. $\mathrm{M}$-opsin staining was detected throughout the retina of the MNU $+\alpha B C$ group, although with a decreased intensity compared with the normal control group. Conversely, no S-opsin staining was detected in any part of the retinas in the $\mathrm{MNU}+\alpha \mathrm{BC}$ group, indicating different sensitivities to $\alpha \mathrm{BC}$ treatment in the cone populations.

Furthermore, another group of mice $(n=10)$ was randomly selected and received an 


\section{Cellular Physiology Cell Physiol Biochem 2018;48:2147-2160

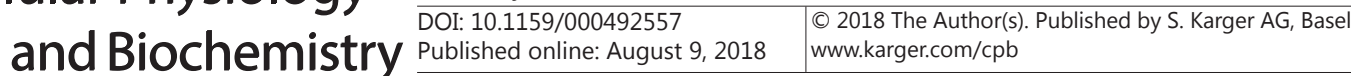

Fig. 6. The mRNA levels of Bax and Caspase-3 in the MNU $+\alpha B C$ group were significantly lower compared with the MNU group $(\mathrm{P}<0.01 ; \mathrm{n}=10)$, whereas the mRNA levels of Bcl-2 and CNTF in the $\mathrm{MNU}+\alpha \mathrm{BC}$ group were significantly compared with the MNU group $(\mathrm{P}<0.01 ; \mathrm{n}=10)$. The mRNA level of BDNF in the MNU $+\alpha B C$ group was not statistically different from that in the MNU group ( $\mathrm{P}>0.05$; $\mathrm{n}$ $=10$; All the values were presented as mean $\pm \mathrm{SD}$; ANOVA analysis followed by Bonferroni's post-hoc analysis was performed, ${ }^{*} \mathrm{P}<0.01$, for differences

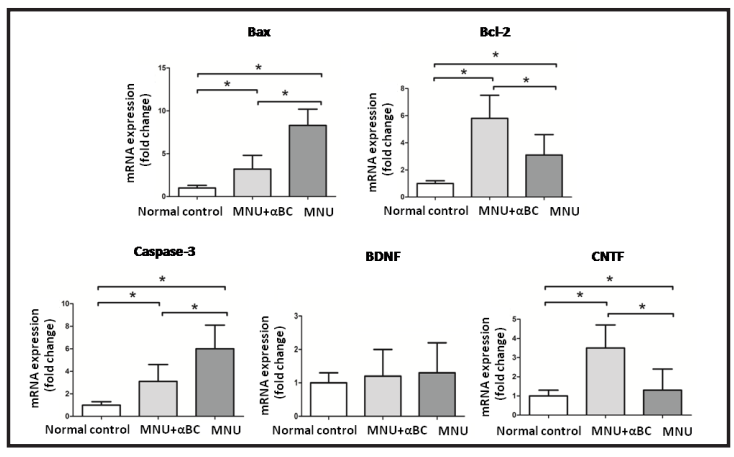
between groups; $n=10$ ).

Fig. 7. Protein levels of Bax and Caspase- 3 in the $\mathrm{MNU}+\alpha \mathrm{BC}$ group were significantly lower compared with the MNU group. Moreover, protein levels of Bcl-2 and CNTF in the MNU $+\alpha B C$ group were significantly higher compared with the MNU group. Additionally, the protein level of BDNF in the MNU $+\alpha B C$ group was not significantly different from that in the MNU group. (All the values were presented as mean \pm SD; ANOVA analysis followed by Bonferroni's post-hoc analysis was performed, ${ }^{*} \mathrm{P}<0.01$, " $\mathrm{P}<0.05$ for differences

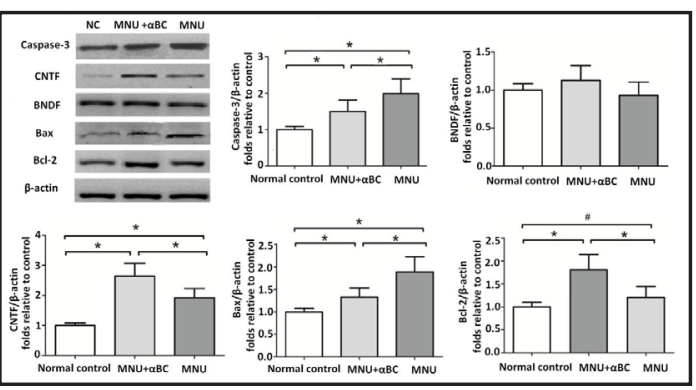
between groups; $\mathrm{n}=10$ ).

Fig. 8. A. $M D A$ concentration in the $M N U+\alpha B C$ group was significantly lower compared with the MNU group. SOD level in the $\mathrm{MNU}+\alpha \mathrm{BC}$ group was significantly higher compared with the MNU group, suggesting that oxygen stress was effectively

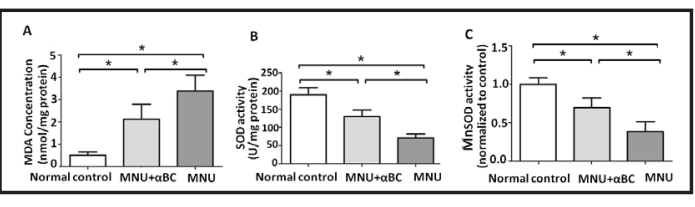
alleviated by $\alpha \mathrm{BC}$ treatment. Mn-SOD level in the MNU group was significantly lower compared with the normal control group, whereas Mn-SOD level in the $\mathrm{MNU}+\alpha \mathrm{BC}$ group was significantly higher compared with the MNU group ( All the values were presented as mean \pm SD; ANOVA analysis followed by Bonferroni's post-hoc analysis was performed, $* \mathrm{P}<0.01$, for differences between groups; $\mathrm{n}=10$ ).

intravitreal injection of heparin $(2 \mu \mathrm{l}, 50 \mathrm{U} / \mathrm{ml}) 2 \mathrm{~h}$ post MNU administration. One week later, these heparin treated mice were subjected to functional evaluation. The mean amplitudes of ERG b-waves in heparin treated mice were not significantly different from those in the MNU group $(P>0.05, \mathrm{n}=10)$. In the optokinetic tests, both visual acuity and contrast sensitivity in the heparin treated group were not significantly different from those in the MNU group $(P>0.05, \mathrm{n}=10)$. Moreover, mean ONL thickness in the heparin treated mice was not significantly different from that in the MNU group $(P>0.05, \mathrm{n}=10)$. Taken together, these findings suggest that intravitreal injection of heparin, a protein unrelated to small heat shock protein, would not affect retinal function or morphology [26]. Alternatively, the aforementioned beneficial effects could be unique to $\alpha \mathrm{BC}$ rather than simply caused by the surgical procedures of intravitreal injection.

\section{Mechanisms underlying the $\alpha B C$ induced protection}

We performed the qRT-PCR to analyse the underlying mechanisms related to the $\alpha B C$ induced effects. The retinal mRNA levels of both Bax and Caspase-3 in the MNU $+\alpha B C$ group were significantly lower than those in the MNU group $(P<0.01 ; \mathrm{n}=10$, Fig. 6). Meanwhile, the mRNA levels of Bcl-2 and CNTF in the MNU $+\alpha B C$ group were significantly higher than 
those in the MNU group $(P<0.01 ; \mathrm{n}=10)$, indicating that neurotrophic and anti-apoptotic mechanisms contributed collectively to the $\alpha B C$ induced protective effects. Of note, the mRNA level of BDNF in the MNU $+\alpha B C$ group was not significantly different from the level in the MNU group $(P>0.05 ; \mathrm{n}=10)$. The protein levels of Bax and Caspase- 3 in the MNU $+\alpha B C$ group were significantly lower than those in the MNU group $(\mathrm{P}<0.01 ; \mathrm{n}=10 ;$ Fig.7), whereas the protein levels of Bcl-2 and CNTF in the MNU $+\alpha B C$ group were significantly higher than that in the MNU group $(P<0.01 ; n=10)$. Additionally, the protein level of BDNF in the $\mathrm{MNU}+\alpha \mathrm{BC}$ group was not significantly different from that in the MNU group $(P>0.05 ; n=10)$. These western blotting data were consisted with the qRT-PCR results. Furthermore, the protein levels of STAT3, phosphorylated STAT3 and JAK in the MNU $+\alpha B C$ group were significantly higher than those in the MNU group $(P<0.01, \mathrm{n}=10$, Fig. 5.B). These findings suggested that JAK/STAT3 signal pathway was activated after $\alpha B C$ treatment. Furthermore, the MDA concentration in the MNU $+\alpha B C$ group was significantly lower than that in the MNU group $(P<0.01 ; \mathrm{n}=10$, Fig. 8.A). Conversely, the SOD level in the MNU $+\alpha B C$ group was significantly higher $(P<0.01$; $\mathrm{n}=10$, Fig. 8.B), suggesting that oxygen stress was effectively alleviated by $\alpha \mathrm{BC}$ treatment. The Mn-SOD level in the MNU group was significantly lower than the normal controls $(P<$ $0.01 ; \mathrm{n}=10 ;$ Fig. 8.C), whereas the Mn-SOD level in the MNU $+\alpha B C$ group was significantly higher than that in the MNU group $(P<0.01 ; \mathrm{n}=10)$, suggesting that $\alpha \mathrm{BC}$ conferred critical protection against mitochondrial insults during MNU induced photoreceptor apoptosis.

\section{Discussion}

Crystalline, a member of the small heat shock protein family, is a major constitute of the lens. In addition to acting as a static structural protein within the ocular compartments, crystalline confers anti-apoptotic and nutritional effects [27]. There is general agreement that crystallin is necessary to maintain retinal homeostasis. Following traumatic or toxic assault of the retina, endogenous $\alpha B C$ is released from the ocular lens, thereby maintaining high intravitreal concentrations [14]. Notably, endogenous $\alpha B C$ is also secreted by human RPE cells in the form of exosomes, which are released into the extracellular matrix and absorbed by adjacent retinal cells [28]. Theses adaptive activities provide a primary defense against undesirable outcomes of cellular stress, highlighting the possibility that $\alpha \mathrm{BC}$ might be used therapeutically $[29,30]$. To date, the $\alpha B C$ induced protection has been demonstrated in various cell cultures including RPE cells, astrocytes, endothelial cells, and pericytes [31]. A pioneering therapeutic study showed that intravitreal delivered $\beta$-crystallins or $\gamma$-crystallins exerted nutritional effects on the RGCs after optic nerve crush. However, deposition of the exogenous $\alpha$-crystalline within the vitreous cavity failed to produce similar cytoprotective effects [24]. Therefore, we injected the $\alpha \mathrm{BC}$ into the vitreous cavity of MNU administered mice to verify its feasibility for RP therapy.

Here, we showed that intravitreal delivered $\alpha B C$ could ameliorate MNU induced photoreceptor degeneration. Since traditional systemic administration has limitations such as rapid protein clearance and unexpected off-target effects, targeted intravitreal delivery can warrant higher concentrations of $\alpha \mathrm{BC}$ in the intended tissue. The optokinetic behavioral test and ERG examination suggested that visual function of the $\mathrm{MNU}+\alpha \mathrm{BC}$ group is efficiently rescued. Subsequent OCT analysis showed that the main retinal architecture of the MNU $+\alpha B C$ group was preserved by $\alpha \mathrm{BC}$ treatment. The size of lesion was markedly reduced by $\alpha \mathrm{BC}$ treatment as shown in the fundus photographs. In particular, the immunostaining study suggested that photoreceptor populations had different sensitivities to $\alpha B C$. Taken together, our findings highlight a unique therapeutic potential for $\alpha B C$. In previous studies, $\alpha B C$ has been used to treat several ophthalmological diseases, such as optic nerve crush, autoimmune uveoretinitis, diabetic retinopathy, corneal neovascularization, and selenite induced cataracts [11]. In the retina, $\alpha \mathrm{BC}$ can counteract the sodium iodate induced photoreceptor degeneration [32]. All these findings highlight the opportunity to develop a promising therapeutic tool for retinopathy.

\section{KARGER}




\section{Cellular Physiology Cell Physiol Biochem 2018;48:2147-2160 \\ \begin{tabular}{l|l} 
and Biochemistry Published online: August 9, 2018 & $\begin{array}{l}\text { C } 2018 \text { The Author(s). Published by S. Karger AG, Basel } \\
\text { www.karger.com/cpb }\end{array}$
\end{tabular} \\ Tao et al.: Ab-Crystallin Ameliorates Photoreceptor Degeneration.}

$\alpha B C$ is a potent anti-apoptotic protein that interacts with apoptotic mediators. Moreover, prominent $\alpha \mathrm{BC}$ expressions in the mitochondria could prevent activation of the apoptotic cascade $[18,32]$. On the other hand, apoptosis is a pivotal contributory factor to RP. In accordance with this notion, the major attribute of the $\alpha B C$ induced protection appears to be anti-apoptosis. In MNU administered mice, the retinal expressions of Bax and Caspase-3, two critical mediators of apoptosis, were down-regulated after $\alpha \mathrm{BC}$ treatment. On the other hand, the retinal expression of bcl-2, an anti-apoptotic factor was up-regulated after $\alpha B C$ treatment. These findings suggest that adjustment of the apoptotic threshold can serve as a protective strategy against RP. $\alpha \mathrm{BC}$ can also translocate to the mitochondrial voltagedependent anion channels, thereby alleviating oxidative stress [14]. Additionally, $\alpha \mathrm{BC}$ can protect the retinal cells against excessive ER stress, which is associated with bursts of reactive oxygen species(ROS) and superoxide dismutase depletion [17]. This notion is reinforced by the fact that silencing of $\alpha \mathrm{BC}$ exacerbates oxidative stress. whereas the over-expression has the opposite effect [33]. In the present study, we have shown that $\alpha B C$ treatment enhances retinal SOD activity and reduces MDA concentration in MNU administered mice. Furthermore, the CNTF expression was up-regulated and its major downstream signaling pathway (JAK/ STAT3) was activated after $\alpha \mathrm{BC}$ treatment. These alterations would provide essential nutritional support for photoreceptors. Independently, another study also suggested that exogenous $\beta$ crystalline enhanced the CNTF expression in retinal astrocytes. JAK/STAT3 activation was involved in this beneficial effects and was proposed as a necessary prerequisite for axon regeneration in RGCs [24]. Collectively, these findings raise the possibility that $\alpha B C$ supplementation might afford protection against photoreceptor degeneration. $\alpha \mathrm{BC}$ is also reported to be anti-inflammatory owing to its chaperone activity to bind with inflammatory molecules such as Toll-like receptors, interferon- $\gamma$, nuclear factor- $k \beta$, tumor necrosis factor- $\alpha$, interleukin-6 [34]. Intravenous administration of $\alpha B C$ alleviates the inflammatory response in endophthalmitis and uveitis models $[31,35]$. On the other hand, chronic inflammation is recognized as an etiological factor in RP pathogenesis. Inflammatory cells and proteins in the vitreous body increase significantly in RP patients [36]. Preclinical trials targeting activated microglia in the outer retina have already demonstrated impressive benefits in RP animal models [37]. In this context, it is highly plausible that the anti-apoptotic activity of $\alpha B C$ might act together with its anti-inflammatory activity to cause favorable effects. Further investigations are needed to unravel the detailed role of $\alpha \mathrm{BC}$ during the neuroprotection.

Mitochondria are considered as the central regulators of apoptotic cell death that mediate stress signals between critical organelles. Impaired mitochondrial function is closely associated with apoptosis of retinal neuron $[17,29]$. In this study, the $\alpha B C$ could enhance the Mn-SOD activity in the MNU $+\alpha B C$ group, suggesting that $\alpha \mathrm{BC}$ confers critical mitochondrial protection during photoreceptor apoptosis. Moreover, the mitochondrial ROS assay is another method to evaluate the severity of oxidative stress. This examination reliably reflects the $\alpha \mathrm{BC}$ induced effects on mitochondrial function. It is highly possible that $\alpha B C$ would also affect the mitochondrial ROS activity in MNU administered mice. Further studies are necessary to quantify the $\alpha \mathrm{BC}$ induced effects on mitochondrial ROS.

More than 100 gene mutations that initiate RP have been characterized. These mutations are restricted exclusively to the rod photoreceptors which are responsible for night vision. However, apoptosis of the rods is followed by secondary cone loss [3]. Since cones are not directly affected by primary mutations, the causes of cone loss as well as protection represent the main challenges in RP research [38]. As long as the cones are rescued, RP patients would be able to function well under bright conditions and lead relatively normal lives despite the primary loss of rods $[39,40]$. To our knowledge, this is the first study to report the $\alpha \mathrm{BC}$ induced protection on cones. Accordingly, it would be of great value to develop a cone rescuing reagent to maintain daily vision of RP patients. Specifically, the Mcone photoreceptors, rather than the S-cone photoreceptors, in MNU administered mice are efficiently rescued by $\alpha \mathrm{BC}$ treatment. In mouse retina, the cone photoreceptors are not evenly distributed, and each subtype forms a gradient across the retinal regions: S-cone photoreceptors are mainly distributed in the inferior hemisphere, particular the nasal- 


\section{Cellular Physiology Cell Physiol Biochem 2018;48:2147-2160 \begin{tabular}{l|l|l} 
DOI: 10.1159/000492557 & and Biochemistry Published online: August 9, 2018 & $\begin{array}{l}\text { O 2018 The Author(s). Published by S. Karger AG, Basel } \\
\text { www.karger.com/cpb }\end{array}$
\end{tabular}}

inferior quadrant; M-cone photoreceptors are mainly distributed in superior hemisphere $[21,41]$. On the other hand, the focal photoreceptors show different vulnerabilities to MNU toxicity and displayed positional asymmetry among the retinal quadrants [42-44]. These photoreceptors in the nasal-inferior quadrant are most vulnerable to MNU toxicity and the impairment in this region is most severe. Conversely, the photoreceptors in the temporalinferior quadrant are most resistant to MNU toxicity and the impairment in this region is least severe. Therefore, the relative number and distribution of photoreceptor populations across retinal regions closely correlate with the differences in "protective effects". It may be more difficult to rescue with therapeutic agents the S-cones which are concentrated in the nasal-inferior quadrant. Future studies are necessary to verify this proposal.

In conclusion, intravitreal delivery of $\alpha \mathrm{BC}$ can alleviate photoreceptor degeneration in $\mathrm{MNU}$ administered mice. $\alpha \mathrm{BC}$ induced protection might stem from anti-apoptotic, anti oxidative and nutritional attributes. Our findings highlight the possibility that intravitreal delivery of $\alpha \mathrm{BC}$ might be a promising strategy for the treatment of RP. Further studies on the dosage regimens, delivery route, and intervention time points would be of great importance for $\alpha \mathrm{BC}$ therapy.

\section{Acknowledgements}

This work was supported by the National key research and development program(2018YFA0107303); National Natural Science Foundation of China (No.81600767, 81770887, and 81501063).

\section{Disclosure Statement}

All authors declared to have no conflict of interests.

\section{References}

1 Strong S, Liew G, Michaelides M: Retinitis pigmentosa-associated cystoid macular oedema: pathogenesis and avenues of intervention. Br J Ophthalmol 2017;101:31-37.

-2 Huckfeldt RM, Comander J: Management of Cystoid Macular Edema in Retinitis Pigmentosa. Semin Ophthalmol 2017; 32:43-51.

-3 Wert KJ, Lin JH, Tsang SH: General pathophysiology in retinal degeneration. Dev Ophthalmol 2014;53:33-43.

4 Li YJ, Jiang Q, Cao GF, Yao J, Yan B: Repertoires of autophagy in the pathogenesis of ocular diseases. Cell Physiol Biochem 2015; 35:1663-1676.

5 Hamel C: Retinitis pigmentosa. Orphanet J Rare Dis 2006;11;1:40.

6 Wang Y, Yin Z, Gao L, Sun D, Hu X, Xue L, Dai J, Zeng Y, Chen S, Pan B, Chen M, Xie J, Xu H: Curcumin Delays Retinal Degeneration by Regulating Microglia Activation in the Retina of rd1 Mice. Cell Physiol Biochem 2017;44:479-493.

7 Shintani K, Shechtman DL, Gurwood AS: Review and update: current treatment trends for patients with retinitis pigmentosa. Optometry 2009; 80:384-401.

-8 Tsubura A, Yoshizawa K, Kuwata M, Uehara N: Animal models for retinitis pigmentosa induced by MNU; disease progression, mechanisms and therapeutic trials. Histol Histopathol 2010;25:933-944.

-9 Tao Y, Chen T, Liu Z, Xu W, Qin L, Peng G, Huang YF: Integrating Topographic Measures to Explore the Protective Effects of Peonidin Against the N-Methyl-N-Nitrosourea Induced Photoreceptor Degeneration. Cell Physiol Biochem 2016; 38:893-908.

10 Emoto Y, Yoshizawa K, Uehara N, Kinoshita Y, Yuri T, Shikata N, Tsubura A: Curcumin suppresses N-methyl-Nnitrosourea-induced photoreceptor apoptosis in Sprague-Dawley rats. In vivo 2013;27:583-590.

11 Rösch S, Werner C, Müller F, Walter P: Photoreceptor degeneration by intravitreal injection of N-methyl-Nnitrosourea (MNU) in rabbits: a pilot study. Graefes Arch Clin Exp Ophthalmol 2017; 255:317-331. 


\section{Cellular Physiology Cell Physiol Biochem 2018;48:2147-2160 \begin{tabular}{l|l} 
and Biochemistry Published online: August 9, 2018 & $\begin{array}{l}\text { C) } 2018 \text { The Author(s). Published by S. Karger AG, Basel } \\
\text { www.karger.com/cpb }\end{array}$ \\
\hline
\end{tabular}}

12 Ram H. Nagaraj, Rooban B. Nahomi, Niklaus H. Mueller, Cibin T. Raghavan, David A. Ammar, J. Mark Petrash: Therapeutic potential of a-crystallin. Biochimica et Biophysica Acta 2016; 1860:252-257.

-13 G. Kappe, E. Franck, P. Verschuure,W.C. Boelens, J.A. Leunissen, W.W. de Jong: The human genome encodes 10 alpha-crystallin-related small heat shock proteins: HspB1-10 Cell Stress Chaperones 2003;8:53-61.

-14 T. Iwaki, A. Kume-Iwaki, J.E. Goldman: Cellular distribution of alpha B-crystallin in non-lenticular tissues, J. Histochem. Cytochem 1990; 38:31-39.

15 Solon Thanos, Michael R.R. Bohm, Melissa Meyer zu Horste, Verena Prokosch-Willing, Maren Hennig, Dirk Bauer , Arndt Heiligenhaus: Role of crystallins in ocular neuroprotection and axonal regeneration. Progress in Retinal and Eye Research 2014; 42:145-161.

-16 Johnson PT, Brown MN, Pulliam BC, Anderson DH, Johnson LV: Synaptic pathology, altered gene expression, and degeneration in photoreceptors impacted by drusen. Invest. Ophthalmol. Vis. Sci 2005;46: 4788-4795.

17 Umeda S, Suzuki MT, Okamoto H, Ono F, Mizota A, Terao K, Yoshikawa Y, Tanaka Y, Iwata T: Molecular composition of drusen and possible involvement of anti-retinal autoimmunity in two different forms of macular degeneration in cynomolgus monkey (Macaca fascicularis). FASEB J 2005;19:1683-1685.

18 Dou G, Sreekumar PG, Spee C, He S, Ryan SJ: Deficiency of alphaB crystallin augments ER Stress-induced apoptosis by enhancing mitochondrial dysfunction. Free Radic Bio Med 2012; 53: 1111-1122.

19 Hamann, S. Metrailler, S. Schorderet, D.F. Cottet, S: Analysis of the cytoprotective role of alpha-crystallins in cell survival and implication of the alphaA-crystallin C-terminal extension domain in preventing Baxinduced apoptosis. PloS One 2013.;8:e55372.

-20 Wang YH, Wang DW, Wu N, Wang Y, Yin ZQ: Alpha-Crystallin promotes rat axonal regeneration through regulation of RhoA/rock/cofilin/MLC signaling pathways. J. Mol. Neurosci 2012:46;138-144.

-21 Skeie, J.M. Mahajan, V.B: Proteomic interactions in the mouse vitreous-retina complex. PLoS One 2013;8: e82140.

22 Tao Y, Chen T, Fang W, Peng G, Wang L, Qin L, Liu B, Fei Huang Y: The temporal topography of the N-Methyl$\mathrm{N}$-nitrosourea induced photoreceptor degeneration in mouse retina. Sci Rep 2015; 21;5:18612.

23 Gao Y, Deng XG, Sun QN, Zhong ZQ: Ganoderma spore lipid inhibits N-methyl-N-nitrosourea-induced retinal photoreceptor apoptosis in vivo. Exp Eye Res 2010;90: 397-404.

24 Tsubura A, Lai YC, Miki H, Sasaki T, Uehara N, Yuri T, Yoshizawa K: Review: Animal models of N-Methyl-Nnitrosourea-induced mammary cancer and retinal degeneration with special emphasis on therapeutic trials. In vivo $2011 ; 25: 11-22$.

25 Fischer D, Hauk TG, Müller A, Thanos S: Crystallins of the beta/gammasuperfamily mimic the effects of lens injury and promote axon regeneration. Mol Cell Neurosci 2008;37:471-479.

26 Tao Ye, Jixue Yang, Zhao Ma, Zhongjun Yan,Yunqin Wang, Zhen Yang, Yi Fei Huang: The vigabatrin induced retinal toxicity is associated with photopic exposure and taurine deficiency: an in vivo study. Cellular Physiology and Biochemistry 2016;40:831-846.

27 Machida S, Chaudhry P, Shinohara T, Singh DP, Reddy VN, Chylack LT Jr, Sieving PA, Bush RA: Lens epitheliumderived growth factor promotes photoreceptor survival in light-damaged and RCS rats. Invest Ophthalmol Vis Sci 2001;42:1087-1095.

28 S.P. Bhat, C.N. Nagineni: Alpha B subunit of lens-specific protein alpha-crystallin is present in other ocular and non-ocular tissues. Biochem Biophys Res Commun1989;158:319-325.

-29 Sreekumar PG, Kannan R, KitamuraM, Spee C, Barron E: AlphaB crystallin is apically secreted within exosomes by polarized human retinal pigment epithelium and provides neuroprotection to adjacent cells. PLoS ONE 2010;5:e12578.

-30 Kannan R, Sreekumar PG, Hinton DR: Alpha Crystallins in the Retinal Pigment Epithelium and Implications for the Pathogenesis and Treatment of Age-Related Macular Degeneration. Biochim Biophys Acta 2016;1860:258-268.

-31 Zhang J, Zhao X, Cai Y, Li Y, Yu X, Lu L: Protection of Retina by Mini- a A in NaIO3-Induced Retinal Pigment Epithelium Degeneration Mice. Int. J. Mol. Sci 2015;16:1644-1656.

-32 Ram Kannan, Parameswaran G. Sreekumar, and David R. Hinton: Novel roles for a -crystallins in retinal function and disease. Prog Retin Eye Res 2012;31:576-604.

-33 Zhou P, Kannan R, Spee C, Sreekumar PG, Dou G, et al.: Protection of Retina by aB Crystallin in Sodium Iodate Induced Retinal Degeneration. PLoS ONE 2014;9:e98275. 


\section{Cellular Physiology Cell Physiol Biochem 2018;48:2147-2160 \begin{tabular}{l|l|l} 
DOI: 10.1159/000492557 & and Biochemistry $\begin{array}{l}\text { P } 2018 \text { The Author(s). Published by S. Karger AG, Basel } \\
\text { www.karger.com/cpb }\end{array}$ \\
\hline
\end{tabular} \\ Tao et al.: Ab-Crystallin Ameliorates Photoreceptor Degeneration.}

-34 Yaung J, Kannan R, Wawrousek EF, Spee C, Sreekumar PG, Hinton DR: Exacerbation of retinal degeneration in the absence of alpha crystallins in an in vivo model of chemically induced hypoxia. Exp Eye Res 2008;86:355365.

-35 Arac A, Brownell SE, Rothbard JB, Chen C, Ko RM, Pereira MP, Albers GW, Steinman L, Steinberg GK: Systemic augmentation of alphaB-crystallin provides therapeutic benefit twelve hours poststroke onset via immune modulation. Proc Natl Acad Sci USA 2011;108:13287-13292.

-36 Whiston EA, Sugi N, Kamradt MC, Sack C, Heimer SR, Engelbert M, Wawrousek EF, Gilmore MS, Ksander BR, Gregory MS: AlphaB-crystallin protects retinal tissue during Staphylococcus aureus-induced endophthalmitis. Infect Immun 2008;76:1781-1790.

-37 Newsome DA, Michels RG: Detection of lymphocytes in the vitreous gel of patients with retinitis pigmentosa. Am J Ophthalmol 1988;105:596-602.

38 Iezzi R, Guru BR, Glybina IV, Mishra MK, Kennedy A, Kannan RM: Dendrimer-based targeted intravitreal therapy for sustained attenuation of neuroinflammation in retinal degeneration. Biomaterials 2012;33:979988.

-39 Daniel S. Narayan, John P. M. Wood, Glyn Chidlow, Robert J. Casson: A review of the mechanisms of cone degeneration in retinitis pigmentosa. Acta Ophthalmol 2016;94:748-754.

40 Remé CE, Grimm C, Hafezi F, Iseli HP, Wenzel A: Why study rod cell death in retinal degenerations and how? Doc Ophthalmol 2003;106:25-29.

-41 Wheaton DH, Hoffman DR, Locke KG, Watkins RB, Birch DG: Biological safety assessment of docosahexaenoic acid supplementation in a randomized clinical trial for X-linked retinitis pigmentosa. Arch Ophthalmol 2003;121:1269-1278.

-42 Boudard D L. Tanimoto N, Huber G, Beck SC, Seeliger MW, Hicks D: Cone loss is delayed relative to rod loss during induced retinal degeneration in the diurnal cone-rich rodent Arvicanthis ansorgei. Neuroscience 2010;169:1815-1830.

43 Ahnelt PK, Kolb H: The mammalian photoreceptor mosaic-adaptive design. Prog Retin Eye Res 2000;19:711777.

44 Szél A, Röhlich P, Caffé AR, van Veen T: Distribution of cone photoreceptors in the mammalian retina. Microsc. Res. Tech 1996;35:445-462. 